P. C. $\mathrm{Hu}$

Nagoya Math. J.

Vol. 120 (1990), 155-170

\title{
HOLOMORPHIC MAPPING INTO ALGEBRAIC VARIETIES OF GENERAL TYPE
}

\author{
PEICHU HU
}

\section{§1. Introduction}

We will study holomorphic mappings

$$
f: M \longrightarrow N
$$

from a connected complex manifold $M$ of dimension $m$ to a projective algebraic manifold $N$ of dimension $n$. Assume first that $N$ is of general type, i.e.

$$
\varlimsup_{k \rightarrow \infty} \frac{\operatorname{dim} H^{0}\left(N, K_{N}^{k}\right)}{k^{n}}>0,
$$

where $K_{N} \rightarrow N$ is the canonical bundle of $N$. If $K_{N}$ is positive, then $N$ is of general type.

In 1971, Kodaira [6] obtained that

Theorem A. Any holomorphic mapping $f: C^{m} \rightarrow N$ has every-where rank less than $n$.

P. Griffiths \& J. King [2], [3] furthermore proved that

THeOREM B. If $M$ is a smooth affine algebraic variety, then any holomorphic mapping $f: M \rightarrow N$ whose image contains an open set is necessarily rational.

In 1977, W. Stoll [6] extended Theorems A, B to parabolic manifolds $M$. To state it, we let $M$ possess a parabolic exhaustion $\tau$ and denote

$$
\nu=d d^{c} \tau, \quad \sigma=d^{c} \log \tau \wedge\left(d d^{c} \log \tau\right)^{m-1} .
$$

For a form $\varphi$ of bidegree $(1,1)$ on $M$, write

$$
A(t, \varphi)=t^{2-2 m} \int_{M[t]} \varphi \wedge \nu^{m-1}, \quad T(r, s ; \varphi)=\int_{s}^{r} \frac{A(t, \varphi)}{t} d t
$$

Received July 10, 1989. 
if the integrals exist, where $M[t]=\left\{x \in M: \tau(x) \leq t^{2}\right\}$. Suppose throughout that $L$ is a positive holomorphic line bundle over $N$ with a hermitian metric $\rho$ along the fibers of $L$ such that the Chern form $c(L, \rho)>0$. The characteristic function of $f$ for $L$ is defined by

$$
T(r, s)=T\left(r, s ; f^{*} c(L, \rho)\right) .
$$

THeorem C. If $M$ is a parabolic manifold and if $F$ is an effective Jacobian section such that

(i) $F$ is dominated by $\tau$ with $Y$ as dominator, there exist positive constants $c_{1}, c_{2}, c_{3}$ such that for $\varepsilon>0$

$$
T(r, s) \leq c_{1} \log Y(r)+c_{2} \operatorname{Ric}_{\tau}(r, s)+c_{3} \varepsilon \log r
$$

with the exception of a set of values $(r)$ of finite measure.

The condition (i) implies $m \geq n=\operatorname{rank} f$ ([8], Lemma 18.1). We remove this restriction (see [4]). To state the generalization of the Theorem $\mathrm{C}$ which we shall prove, we take a positive form $\psi$ of class $C^{\infty}$ and bidegree $(1,1)$ on $N$ and set

$$
\psi_{f}= \begin{cases}f^{*}\left(\psi^{m}\right) & \text { if } m \leq n \\ f^{*}\left(\psi^{n}\right) \wedge \chi & \text { if } m>n\end{cases}
$$

where $\chi$ be a positive $(m-n, m-n)$-form of class $C^{\infty}$ on $M$. Then the form

$$
\chi_{f}=f^{*}\left(\operatorname{Ric} \psi^{n}\right)-\frac{n}{b} \operatorname{Ric} \psi_{f} \quad \text { where } b=\min (m, n),
$$

is well-defined. Take a holomorphic form B of bidegree $(m-1,0)$ on $M$. Define

$$
\begin{aligned}
\ddot{\psi}_{f} & =\ddot{\psi}_{f}(B)=m i_{m-1} f^{*}(\psi) \wedge B \wedge \bar{B}, \\
e_{f} & =e_{f}(\psi)=f^{*}\left(\operatorname{Ric} \psi^{n}\right)-n \operatorname{Ric} \ddot{\psi}_{f},
\end{aligned}
$$

where $i_{m-1}$ is defined in Section 3. Then $\chi_{f}(h \psi)=\chi_{f}(\psi), e_{f}(h \psi)=e_{f}(\psi)$ for positive functions $h$ of class $C^{2}$ on $N$. Define $\eta$ by $\ddot{\psi}_{f}=\eta f^{*}(\psi) \wedge \nu^{m-1}$ and denote

$$
\begin{gathered}
B(r, \eta)=\frac{1}{2} \int_{\partial M[r]} \log \eta \sigma, \\
E_{f}(r, s)=T\left(r, s ; e_{f}\right)+\left.n B(t, \eta)\right|_{s} ^{r},
\end{gathered}
$$


where $\left.B(t)\right|_{s} ^{r}$ means $B(r)-B(s)$. For $\psi=c(L, \rho)$, we obtain that

THEOREM 1. If there exists an effective Jacobian section of $f$ and if rank $f=b=\min (m, n)$, then exist positive constants $c_{1}$ and $c_{2}$ such that for $\varepsilon>0$

$$
c_{1} T(r, s) \leq \mathfrak{n} \operatorname{Ric}_{\imath}(r, s)+E_{f}(r, s)+c_{2} \varepsilon \log r
$$

with the exception of a set of values $(r)$ of finite measure.

CoRollary 2. If $M$ is smooth affine algebraic variety, any non-degenerate holomorphic mapping $f: M \rightarrow N$ with

$$
\varlimsup_{r \rightarrow \infty} \frac{E_{f}(r, s)}{\log r}<\infty
$$

is necessarily rational.

To draw geometrical consequences, here assume that $M$ and $N$ are hermitian manifolds. Relative to the local coordinates $z^{i}$ let

$$
d s_{M}^{2}=\sum_{i, j} h_{i j} d z^{i} d \bar{z}^{j} \quad 1 \leq i, j \leq m
$$

be a positive definite hermitian metric on $M$ with the associated 2 -form

$$
\varphi=\frac{\sqrt{-1}}{2 \pi} \sum_{i, j} h_{i j} d z^{i} \wedge d \bar{z}^{1}
$$

Similarly, let

$$
d s_{N}^{2}=\sum_{k, l} \tilde{h}_{k l} d w^{k} d \bar{w}^{l} \quad 1 \leq k, l \leq n
$$

be a positive definite hermitian metric on $N$, with the local coordinates $w^{k}$, and

$$
\psi=\frac{\sqrt{-1}}{2 \pi} \sum_{k, l} \tilde{h}_{k l} d w^{k} \wedge d \bar{w}^{l}
$$

be the associated 2 -form. Define the function $u$ on $M$ by

$$
\psi_{f}=u \varphi^{m} .
$$

Then we have

$$
\partial \bar{\partial} \log u=\operatorname{Ric}_{M}-\frac{b}{n} f^{*}\left(\operatorname{Ric}_{N}\right)+\frac{2 \pi b \sqrt{-1}}{n} \chi_{f} .
$$

When $m \leq n$, 


$$
u=\frac{\operatorname{det}\left(\hat{h}_{i j}\right)}{\operatorname{det}\left(h_{i j}\right)}
$$

is geometrically the ratio of the volume elements, where

$$
\hat{h}_{i j}=\sum_{k, l} \tilde{h}_{k l} \frac{\partial w^{k}}{\partial z^{i}} \frac{\partial \bar{w}^{l}}{\partial \bar{z}^{j}}
$$

under the mapping $f$. If $m=n$, (15) implies the Chern formula [1]

$$
\frac{1}{2} \Delta \log u=R-\operatorname{Tr}\left(f^{*}\left(\operatorname{Ric}_{N}\right)\right)
$$

where $\Delta$ is the Laplacian in $M$ and $R$ denotes the scalar curvature of $M$.

Let $D_{f}$ be the zero divisor of $\psi_{f}$, which independent of the choices of $\psi$ and $\chi$. Then $\chi_{f}$ determines an element $\left[\chi_{f}\right] \in H_{D R}^{2}\left(M-D_{f}, R\right)$, the de Rham cohomology group of closed $C^{\infty}$ differential forms modulo exact ones. We extend the Chern Theorems [1] on holomorphic mappings of hermitian manifolds of the same dimension to non-equidimensional cases. This includes a non-equidimensional version of the Schwarz lemma, which says that if $M$ is the unit $m$-ball and $N$ is almost einsteinian with $\sqrt{-1} \operatorname{Tr}\left(\chi_{f}\right) \geq 0$, the mapping $f$ does not increase volume.

The author learned about value distribution theory from Mo Ye and Yum-Tong Siu, whom he wishes to thank for sharing their insights with him. Also he would like to thank the referee for his suggestions to correct errors in this paper.

\section{$\S 2$. The Ricci form and proof of the formula (15)}

As usual, we let

$$
d=\partial+\bar{\partial} \quad \text { and } \quad d^{c}=\frac{\sqrt{-1}}{4 \pi}(\bar{\partial}-\partial) .
$$

Then

$$
d d^{c}=\frac{\sqrt{-1}}{2 \pi} \partial \widehat{\hat{o}}
$$

The Chern form of the line bundle $L$ for the hermitian metric $\rho$ is defined by

$$
c(L, \rho)=-d d^{c} \log |s|_{\rho}^{2} \quad \text { on } U
$$

for all open subsets $U$ in $N$ and all $s \in H^{\circ}(U, L)$. Let $\Psi$ be a volume form 
on $N$. This is the same as a metric on the canonical line bundle $K_{N}$, which is denoted by $\rho_{\psi}$. In terms of complex coordinates $w^{1}, \cdots, w^{n}$, such a form is one which can be written

$$
\Psi(w)=\rho(w) \Phi(w) \quad \text { where } \Phi(w)=\prod_{j=1}^{n} \frac{\sqrt{-1}}{2 \pi} d w^{j} \wedge d \bar{w}^{j}
$$

and $\rho$ is real $>0$. In practice one often has

$$
\rho(w)=\lambda(w)|g(w)|^{2 q},
$$

where $g$ is holomorphic not identically zero, $q$ is some fixed rational number $>0$ and $\lambda$ is $C^{\infty}$ and $>0$. We define the Ricci form of $\Psi$ to be the Chern form of this metric $\rho_{\Psi}$ on $K_{N}$, so

$$
\operatorname{Ric} \Psi=c\left(K_{N}, \rho_{\Psi}\right)=d d^{c} \log \rho=d d^{c} \log \lambda,
$$

which is independent of the choice of complex coordinates, and defines a real $(1,1)$-form.

Now we prove the formula (15). It is well known that the Ricci form of $M$ for the metric $d s_{M}^{2}$ is of

$$
\operatorname{Ric}_{M}=-\partial \overline{\hat{o}} \log \operatorname{det}\left(h_{i j}\right) .
$$

Then we have

$$
\operatorname{Ric} \varphi^{m}=d d^{c} \log \operatorname{det}\left(h_{i j}\right)=\frac{1}{2 \pi \sqrt{-1}} \operatorname{Ric}_{M} .
$$

It follows that

$$
\begin{aligned}
\chi_{f} & =f^{*}\left(\operatorname{Ric} \psi^{n}\right)-\frac{n}{b} \operatorname{Ric} \psi_{f} \\
& =f^{*}\left(\frac{1}{2 \pi \sqrt{-1}} \operatorname{Ric}_{N}\right)-\frac{n}{b}\left(d d^{c} \log u+\operatorname{Ric} \varphi^{m}\right)
\end{aligned}
$$

which implies (15) by (19).

For convenience, we let $\chi=1$ if $m \leq n$, so that

$$
\psi_{f}=f^{*}\left(\psi^{b}\right) \wedge \chi
$$

Hence when $m \leq n, u$ is independent of the choice of $\chi$ and of the expression (16). Thus

$$
u=\frac{\operatorname{det}\left(\tilde{h}_{k l}\right)}{\operatorname{det}\left(h_{i \jmath}\right)}\left|\operatorname{det}\left(\frac{\partial w^{k}}{\partial z^{i}}\right)\right|^{2}
$$


if $m=n$. When $m>n, u=u_{x}$ depends on the choice of $\chi$ with

$$
u_{h x}=h u_{x}
$$

where $h$ is a function on $M$. Locally we may choose an orthonormal coframe $\theta_{1}, \cdots, \theta_{m}$ for $M$ such that

$$
d s_{M}^{2}=\sum_{j=1}^{m} \theta_{j} \bar{\theta}_{j} .
$$

It is well-known that $d s_{M}^{2}$ induces an intrinsic connection on $M$ and we let

$$
\Omega_{i j}=\frac{1}{2} \sum_{k, l} R_{i j k l} \theta_{k} \wedge \bar{\theta}_{l}
$$

be the curvature. Then

$$
\operatorname{Ric}_{M}=\sum_{i=1}^{m} \Omega_{i i}=\frac{1}{2} \sum_{k, l} R_{k l} \theta_{k} \wedge \bar{\theta}_{l},
$$

where

$$
R_{k l}=\sum_{i=1}^{m} R_{i i k l}
$$

From them we form the scalar curvature

$$
R=\sum_{k=1}^{m} R_{k k} .
$$

Similarly, let $\omega_{1}, \cdots, \omega_{n}$ be an orthonormal co-frame for $N$ such that

$$
d s_{N}^{2}=\sum_{k=1}^{n} \omega_{k} \bar{\omega}_{k}
$$

and let $S_{i j k l}, S_{i j}$ and $S$ be the curvature tensor, the Ricci tensor and scalar curvature of $N$ respectively. We put

$$
\begin{aligned}
d u & =\sum_{i}\left(u_{i} \theta_{i}+\bar{u}_{i} \bar{\theta}_{i}\right), \\
\partial \bar{\partial} u & =-d \partial u=\sum_{i, j} u_{i j} \theta_{i} \wedge \bar{\theta}_{j} .
\end{aligned}
$$

Then the Laplacian of $u$ is defined by

$$
\Delta u=4 \sum_{i} u_{i i}
$$

If $u>0$, we find

$$
\Delta \log u=\frac{1}{u} \Delta u-\frac{4}{u^{2}} \sum_{i} u_{i} \bar{u}_{i}
$$


Under the mapping $f$ let us set

$$
\omega_{i}=\sum_{j=1}^{m} a_{i j} \theta_{j} \quad 1 \leq i \leq n .
$$

If $u>0$, it follows from (15) that

$$
\frac{1}{2} \Delta \log u=R-\frac{b}{n} \sum_{k, l, i} S_{k l} a_{k i} \bar{a}_{l i}+\frac{2 b}{n} \lambda_{f},
$$

where

$$
\lambda_{f}=2 \pi \sqrt{-1} \operatorname{Tr}\left(\chi_{f}\right) .
$$

When $m=n$, (22) implies (17).

To draw geometrical conclusions we start with some definitions: $f$ is said to be degenerate at $p \in M$, if $u$ vanishes at $p$, totally degenerate if $u$ vanishes identically, volume decreasing or volume increasing according as $u \leq 1$ or $u \geq 1$ for a $\chi$. Proceeding in similar manner as Chern [1], we have

Proposition 3. Let $f: M \rightarrow N$ be a holomorphic mapping, where $M, N$ are hermitian manifolds of dimension $m$ and $n$ respectively, with $M$ compact and $N$ einsteinian. Let $R$ and $S$ be their scalar curvature respectively. Then we have

(1) If $R>0, S \leq 0, \lambda_{f} \geq 0$, then $f$ is totally degenerate.

(2) If $R<0, S \geq 0, \lambda_{f} \leq 0$, then there is a point of $M$ at which $f$ is degenerate.

To obtain an upper bound for the scalar function $u$, Chern impose some conditions on the domain manifold $M$ and the image manifold $N$. The first property is:

$\left(D O_{K}\right) . \quad M$ is exhausted by a sequence of open submanifolds

$$
M_{1} \subset M_{2} \subset M_{3} \subset \cdots \subset M
$$

whose closures $\bar{M}_{\alpha}$ are compact, such that: (1) to each $\alpha=1,2, \cdots$ there is a smooth function $\nu_{\alpha} \geq 0$ defined in $M_{\alpha}$, which satisfies the inequality

$$
\frac{1}{2} \Delta \nu_{\alpha} \leq R+K \exp \left(\nu_{\alpha} / m\right)
$$

where $K$ is a given positive constant; $(2) \nu_{\alpha}\left(p_{\beta}\right) \rightarrow \infty$, if $p_{\beta}$ is a divergent sequence of points in $M_{\alpha}$.

For example, the unit ball $M=D_{1}$ defined by 


$$
r^{2}=z_{1} \bar{z}_{1}+\cdots+z_{m} \bar{z}_{m}<1
$$

in the $m$-dimensional number space $C^{m}$ with coordinates $\left(z_{1}, \cdots, z_{m}\right)$ has the property $\left(D O_{K}\right)$, with

$$
\nu_{\rho}=\log \left(\frac{1-r^{2}}{\rho^{2}-r^{2}}\right)^{2 m}
$$

in the exhaustion submanifolds $D_{\rho}$ of $D_{1}$, where $D_{\rho}$ be defined by $r<\rho$ $(<1)$, and $K=2 m(m+1)$. The unit ball is einsteinian with its scalar curvature $R=-2 m(m+1)$ under the kählerian metric

$$
d s_{M}^{2}=\frac{1}{1-r^{2}} \sum_{k} d z_{k} d \bar{z}_{k}+\frac{4 r^{2}}{\left(1-r^{2}\right)^{2}} \partial r \bar{\partial} r
$$

$\left(I M_{K}\right) . \quad N$ is said to have the property $\left(I M_{K}\right)$ (or almost einsteinian), if

$$
\sum_{i, k} S_{i k} \zeta_{i} \bar{\zeta}_{k} \leq-\frac{K}{n} \sum_{i} \zeta_{i} \bar{\zeta}_{i}, \quad \text { for all } \zeta_{i} .
$$

For the rest of this section we let $m \leq n$. Define

$$
A_{j k}=\sum_{i=1}^{n} a_{i j} \bar{a}_{i k}
$$

Then we have

$$
u=\operatorname{det}\left(A_{j k}\right) .
$$

By Hadamard's well-known determinant inequality we have

$$
\frac{1}{m} \sum_{j, k}\left|A_{j k}\right|^{2} \geq\left|\operatorname{det}\left(A_{j k}\right)\right|^{2 / m}=u^{2 / m} .
$$

Hence Cauchy-Hölder's inequality implies

$$
\left(m^{1 / 2} / n\right) u^{1 / m} \leq \frac{1}{n}\left(\sum_{j, k}\left|A_{j k}\right|^{2}\right)^{1 / 2} \leq \frac{1}{n} \sum_{i, j}\left|a_{i j}\right|^{2} .
$$

It follows from (22) that if $N$ have the property $\left(I M_{K}\right)$ and $u>0$ we have

$$
\frac{1}{2} \Delta \log u \geq R+\left(m^{3 / 2} / n^{2}\right) K u^{1 / m}+\frac{2 m}{n} \lambda_{f} .
$$

Now proceeding in similar manner as Chern [1], we have

Proposition 4. Let $f: M \rightarrow N$ be a holomorphic mapping, where $M$ and $N$ are hermitian manifolds of dimension $m$ and $n$ having the properties 
$\left(D O_{K}\right)$ and $\left(I M_{K_{0}}\right)$ respectively, with $K_{0}=\left(n^{2} / m^{3 / 2}\right) K$ and $m \leq n$. If $\lambda_{f} \geq 0$, then $u \leq \exp \left(\nu_{\alpha}\right)$.

Proposition 5. Let $f: D_{1} \rightarrow N$ be a holomorphic mapping, where $D_{1}$ is the unit m-ball with the standard kähler metric and where $N$ is an $n$-dimensional hermitian einsteinian manifold with scalar curvature $\leq-$ $2 n^{2}(m+1) / m^{1 / 2}$ and $n \geq m$. If $\lambda_{f} \geq 0$, then $f$ is volume-decreasing.

\section{$\S 3$. Notes on parabolic manifolds}

From now on, we will study value distribution on the holomorphic mapping $f: M \rightarrow N$. Let $L_{f} \rightarrow M$ be the pull-back of $L \rightarrow N$ and $s_{f}$ the pull-back of $s \in H^{0}(N, L)$. Then $K_{M} \otimes\left(K_{N f}^{*}\right)$ is called the Jacobian bundle, its holomorphic sections over $M$ are called Jacobian sections. A Jacobian section $F$ is called effective if the set $F^{-1}(0)$ of zeroes is thin, its zero divisor $D_{F}$ is called the ramification divisor of $f$ for $F$. Let $A_{k}^{p}(U)$ be the vector space of forms of class $C^{k}$ and degree $p$ on $U \subset N$. Define

$$
i_{p}=\left(\frac{\sqrt{-1}}{2 \pi}\right)^{p}(-1)^{p(p-1) / 2} p ! .
$$

Then a Jacobian section $F$ operates on forms of degree $2 n$ as follows: Take $\Psi \in A_{k}^{2 n}(U)$ with $\tilde{U}=f^{-1}(U) \neq \varnothing$. Relative to the local coordinates $z^{i}$ and $w^{k}$ of $M$ and $N$ respectively, write

$$
\begin{aligned}
& F=g d z^{1} \wedge \cdots \wedge d z^{m} \otimes\left(\frac{\partial}{\partial w^{1}} \wedge \cdots \wedge \frac{\partial}{\partial w^{n}}\right)_{f} \quad g \in \operatorname{Hol}(\tilde{U}), \\
& \Psi=i_{n} h d w^{1} \wedge \cdots \wedge d w^{n} \wedge d \bar{w}^{1} \wedge \cdots \wedge d \bar{w}^{n} .
\end{aligned}
$$

Then

$$
F[\Psi]=i_{m}(h \circ f)|g|^{2} d z^{1} \wedge \cdots \wedge d z^{m} \wedge d \bar{z}^{1} \wedge \cdots \wedge d \bar{z}^{m} .
$$

If $M$ is Stein and if $f$ has strict $\operatorname{rank} \min (m, n)$, effective Jacobian sections exist (see [8]).

Assume that $\tau$ is a parabolic exhaustion of $M$, i.e., a proper map $\tau$ : $M \rightarrow R^{+}$of class $C^{\infty}$ which satisfies

$$
\left\{\begin{array}{l}
d d^{c} \log \tau \geq 0, \\
\left(d d^{c} \tau\right)^{m} \neq 0 \text { but }\left(d d^{c} \log \tau\right)^{m} \equiv 0 \\
M[0] \text { has measure zero. }
\end{array}\right.
$$

For any regular value $r$ of $\tau$, then 


$$
\mathfrak{c}=\int_{\partial M[r]} \sigma
$$

is a constant. Take a positive form $\Omega$ of degree $2 \mathrm{~m}$ and class $C^{2}$ on $M$. Define $v$ by $\nu^{m}=v \Omega$. The Ricci function of $\tau$ is defined by

$$
\operatorname{Ric}_{\tau}(r, s)=T(r, s ; \operatorname{Ric} \Omega)+\left.B(t, v)\right|_{s} ^{r},
$$

which does not depend on the choice of $\Omega$. Let $D$ be a divisor on $M$ and set $D[r]=D \cap M[r]$. We define

$$
\begin{aligned}
& n(t, D)=t^{2-2 m} \int_{D[t]} \nu^{m-1}, \\
& N(r, s ; D)=\int_{s}^{r} n(t, D) \frac{d t}{t} .
\end{aligned}
$$

If we define $v$ by $\nu^{m}=v F[\Psi]$ for an effective Jacobian section $F$ and a positive volume form $\Psi$ of class $C^{\infty}$ and degree $2 n$ on $N$, then

$$
\operatorname{Ric}_{\tau}(r, s)=T\left(r, s ; f^{*}(\operatorname{Ric} \Psi)\right)+\left.B(t, v)\right|_{s} ^{r}+N\left(r, s ; D_{F}\right)
$$

(For a detailed proof see [8] Theorem 15.5).

Take an effective Jacobian section $F$ and a positive form $\psi$ of class $C^{\infty}$ and bidegree $(1,1)$ on $N$. Define $u_{0}$ and $u_{1}$ by

$$
\nu^{m}=u_{0} \ddot{\psi}_{f}, \quad \nu^{m}=u_{1} F\left[\psi^{n}\right] .
$$

By the definitions of $\eta$ and $\ddot{\psi}_{f}$, we have

$$
\nu^{m}=u_{0} \eta f^{*}(\psi) \wedge \nu^{m-1} .
$$

Let $D_{f}$ be the zero divisor of $\ddot{\psi}_{f}$. Then

$$
S_{f}(r, s)=N\left(r, s ; D_{F}\right)-\mathfrak{n} N\left(r, s ; D_{f}\right)+\left.B\left(t, \frac{u_{1}}{u_{0}^{n}}\right)\right|_{s} ^{r}
$$

is defined such that

$$
E_{f}(r, s)+S_{f}(r, s)=(1-n) \operatorname{Ric}_{\tau}(r, s)+\left.n B(t, \eta)\right|_{s} ^{r} .
$$

In fact, the form $\ddot{\psi}_{f}$ determines a section $s_{f}$ of $K_{M}$ such that $\ddot{\psi}_{f}=\left|s_{f}\right|_{\rho}^{2} \Omega$ for a volume form $\Omega$ and a hermitian metric $\rho$ along the fibers of $K_{M}$. Then by Green Residue Theorem [9]

$$
T\left(r, s ; d d^{c} \log \left|s_{f}\right|_{\rho}^{2}\right)+N\left(r, s ; D_{f}\right)=B\left(t,\left|s_{f}\right|_{\rho}^{2}\right)_{s}^{r}
$$

for all regular values $s$ and $r$ of $\tau$ with $0<s<r$. Since 


$$
\operatorname{Ric} \ddot{\psi}_{f}=d d^{c} \log \left|s_{f}\right|_{\rho}^{2}+\operatorname{Ric} \Omega
$$

we have

$$
\begin{aligned}
\operatorname{Ric}_{\tau}(r, s) & =T(r, s ; \operatorname{Ric} \Omega)+\left.B\left(t, u_{0} \cdot\left|s_{f}\right|_{\rho}^{2}\right)\right|_{s} ^{r} & & (\text { by }(31)), \\
& =T\left(r, s ; \operatorname{Ric} \ddot{\psi}_{f}\right)+N\left(r, s ; D_{f}\right)+\left.B\left(t, u_{0}\right)\right|_{s} ^{r} & & (\text { by }(36)) .
\end{aligned}
$$

It follows from (32) that

$$
\operatorname{Ric}_{\tau}(r, s)=T\left(r, s ; f^{*}\left(\operatorname{Ric} \psi^{n}\right)\right)+\left.B\left(t, u_{1}\right)\right|_{s} ^{r}+N\left(r, s ; D_{F}\right) .
$$

Multiply (37) by $\mathfrak{n}$ and minus (38) to obtain (35).

Let $D$ be a divisor given by the zeroes of a holomorphic section $\alpha \epsilon$ $H^{0}(N, L)$. Since $\alpha$ and $\lambda \alpha(\lambda \neq 0)$ define the same divisor and $N$ is compact, we shall assume that $|\alpha(x)|_{\rho} \leq 1$ for $x \in N$, i.e., the metric $\rho$ is distinguished. Assume that $\alpha_{f} \neq \equiv 0$. The proximity form is defined by

$$
m(r, D)=B\left(r,\left|\alpha_{f}\right|^{-2}\right) \geq 0 .
$$

Then we have F. M. T. for any effective divisor (see [3], [8])

$$
N\left(r, s ; D_{f}^{\alpha}\right)+\left.m(t, D)\right|_{s} ^{r}=T(r, s),
$$

where $D_{f}^{\alpha}$ be the divisor of $\alpha_{f} \in H^{0}\left(M, L_{f}\right)$.

The following Lemma is well-known (see Nevanlinna [7]):

LEMMA 6. Let $h(r) \geq 0, g(r) \geq 0$ and $\alpha(r)>0$ be increasing continuous functions of $r$ where $g^{\prime}(r)$ is continuous and $h^{\prime}(r)$ is piecewise continuous. Suppose moreover that $\int^{\infty}(d r / \alpha(r))<\infty$. Then

$$
h^{\prime}(r) \leq g^{\prime}(r) \alpha(h(r))
$$

except for a union of intervals $I \subset R^{+}$such that $\int_{I} d g<\infty$.

We use the notation

$$
\|_{\varepsilon} a(r) \leq b(r)
$$

to mean that the stated inequality holds except on an open set $I \subset \boldsymbol{R}^{+}$ such that $\int_{I} r^{\varepsilon} d r<\infty$ for $\varepsilon>0$.

LeMma 7. Let $\varphi \geq 0$ be a form of bidegree $(1,1)$ on $M$ such that $T(r, s ; \varphi)$ exists. Let $u \geq 0$ be a function on $M$ such that

$$
u \nu^{m} \leq \varphi \wedge \nu^{m-1} \text {. }
$$


Then

$$
\|_{\varepsilon} B(r, u) \leq \frac{\mathfrak{c}}{2}\{(1+2 \varepsilon) \log T(r, s ; \varphi)+4 \varepsilon \log r\} .
$$

Proof. Define

$$
\hat{B}(r, u)=\frac{1}{\mathfrak{c}} \int_{\partial M[r]} u \sigma
$$

Since

$$
\begin{aligned}
0 & \leq r^{2 m-2} A(r, u \nu)=m \int_{M[r]} u \tau^{m-1} d \tau \wedge \sigma=2 m \int_{0}^{r}\left\{\int_{\partial M[t]} u \sigma\right\} t^{2 m-1} d t \\
& =2 m c \int_{0}^{r} \hat{B}(t, u) t^{2 m-1} d t \leq r^{2 m-2} A(r, \varphi)
\end{aligned}
$$

$\hat{B}(t, u)$ exists for almost all $t>0$. Now

$$
\frac{2}{\mathfrak{c}} B(r, u)=\frac{1}{\mathfrak{c}} \int_{\partial M[r]} \log u \sigma \leq \log \hat{B}(r, u)
$$

implies

$$
\begin{aligned}
H(r) & =\int_{s}^{r} t^{1-2 m} d t \int_{0}^{t} r^{2 m-1} \exp \left(\frac{2}{\mathfrak{c}} B(r, u)\right) d r \\
& \leq \int_{s}^{r} t^{1-2 m} d t \int_{0}^{t} r^{2 m-1} \hat{B}(r, u) d r \\
& =\frac{1}{2 m c} \int_{s}^{r} A(t, u \nu) \frac{d t}{t}=\frac{1}{2 m c} T(r, s ; u \nu) \leq \frac{1}{2 m c} T(r, s ; \varphi) .
\end{aligned}
$$

Taking $h(r)=H(r), g(r)=r^{1+\varepsilon} /(1+\varepsilon), \alpha(r)=r^{\lambda}$ with $\varepsilon>0$ and $\lambda>1$, we obtain from Lemma 6 that

$$
\begin{aligned}
\|_{\varepsilon} H^{\prime}(r) & =r^{1-2 m} \int_{0}^{r} r^{2 n-1} \exp \left(\frac{1}{c} B(r, u)\right) d r \leq r^{\varepsilon}(h(r))^{2} \\
& \leq r^{\varepsilon}(T(r, s ; \varphi) /(2 m c))^{2} .
\end{aligned}
$$

Keeping the same $\alpha$ and $g$ and taking $h(r)=r^{2 m-1} H^{\prime}(r)$, we find

$$
\begin{aligned}
& \|_{\varepsilon} r^{2 m-1} \exp \left(\frac{2}{\mathfrak{c}} B(r, u)\right)=\frac{d}{d r}\left(r^{2 m-1} \frac{d H}{d r}\right) \leq r^{\varepsilon}\left(r^{2 m-1} \frac{d H}{d r}\right)^{\lambda} \\
& \leq r^{\varepsilon}\left\{r^{\varepsilon+2 m-1}(T(r, s ; \varphi) /(2 m \mathfrak{c}))^{\lambda}\right\}^{\lambda}
\end{aligned}
$$

which implies

(40) $\quad \|_{\varepsilon} B(r, u) \leq \frac{\mathcal{c}}{2}\left\{\lambda^{2} \log T(r, s ; \varphi)+(\lambda(\varepsilon+2 m-1)+(\varepsilon+1-2 m)) \log r\right.$

$$
\left.-\lambda^{2} \log (2 m c)\right\} \text {. }
$$


Take $0<\delta<\min (1, \varepsilon)$ such that $\varepsilon(4+\delta)+\delta(2 m-1)<6 \varepsilon$. Let $\lambda=1+$ $\delta / 2$. Then $\lambda^{2}<1+2 \varepsilon$ and

$$
\lambda(\varepsilon+2 m-1)+\varepsilon+1-2 m=\frac{1}{2}\{\varepsilon(4+\delta)+\delta(2 m-1)\}<3 \varepsilon .
$$

Hence Lemma 7 follows if $r$ is large enough.

q.e.d.

\section{$\S 4$. Holomorphic maps into algebraic varieties of general type}

Proof of Theorem 1. By Kobayashi-Ochiai [5] and Kodaira [6], an integer $p \in N$ exists such that $L^{p}$ is ample and $k \in N$ exists such that $H^{0}(N, I)$ has positive dimension with $I=K_{N}^{k} \otimes\left(L^{p}\right)^{*}$. Take $\alpha \in H^{0}(N, I)$. Let $D_{f}^{\alpha}$ be the divisor of $\alpha_{f} \in H^{0}\left(M, I_{f}\right)$ and let $\hat{\rho}$ be a distinguished hermitian metric along the fibers of $I$. Then (39) implies

$$
T\left(r, s ; f^{*} c(I, \hat{\rho})\right)=N\left(r, s ; D_{f}^{\alpha}\right)+\left.m(t, D)\right|_{s} ^{r} .
$$

A form $\Psi>0$ of class $C^{\infty}$ and degree $2 n$ exists such that $\operatorname{Ric} \Psi=c\left(K_{N}, \rho_{\Psi}\right)$ and $\hat{\rho}=\left(\rho_{\Psi}\right)^{k} \otimes\left(\rho^{*}\right)^{p}$. Hence

$$
c(I, \hat{\rho})=k \operatorname{Ric} \Psi-p c(L, \rho)
$$

which implies

$$
k T\left(r, s ; f^{*}(\operatorname{Ric} \Psi)\right)-\left.m(t, D)\right|_{s} ^{r}=p T(r, s)+N\left(r, s ; D_{f}^{\alpha}\right) .
$$

A function $v \geq 0$ of class $C^{\infty}$ exists on $M-F^{-1}(0)$ such that $\nu^{m}=v F[\Psi]$ and such that

$$
\operatorname{Ric}_{\tau}(r, s)=N\left(r, s ; D_{F}\right)+\left.B(t, v)\right|_{s} ^{r}+T\left(r, s ; f^{*}(\operatorname{Ric} \Psi)\right)
$$

from (32), where $F$ is an effective Jacobian section of $f$. Define $\tilde{\xi}=$ $\left|\alpha_{f}\right|_{\hat{\rho}}^{2 / k} v^{-1}$. Then

$$
\begin{aligned}
\operatorname{Ric}_{\imath}(r, s)+\left.B(t, \tilde{\zeta})\right|_{s} ^{r} & =N\left(r, s ; D_{F}\right)+T\left(r, s ; f^{*}(\operatorname{Ric} \Psi)\right) \\
-\left.\frac{1}{k} m(t, D)\right|_{s} ^{r} & =N\left(r, s ; D_{F}\right)+\frac{1}{k} N\left(r, s ; D_{f}^{\alpha}\right)+\frac{p}{k} T(r, s) .
\end{aligned}
$$

Therefore

$$
\mathfrak{n} N\left(r, s ; D_{f}\right)+\frac{p}{k} T(r, s) \leq \operatorname{Ric}_{\tau}(r, s)-S_{f}(r, s)+\left.B(t, \zeta)\right|_{s} ^{r},
$$

where $\zeta=u_{1} u_{0}^{-n \tilde{\zeta}}$ and

$$
\psi=c(L, \rho)
$$


Define $\hat{\Psi}=|\alpha|_{\hat{\rho}}^{2 / k} \Psi$. Then

$$
F[\hat{\Psi}]=\left|\alpha_{f}\right|_{\hat{\rho}}^{2 / k} F[\Psi]=\tilde{\check{c}} \nu^{m} .
$$

Since $\hat{\Psi}$ is continuous and $c(L, \rho)>0$, a constant $\gamma_{1}>0$ exists such that $\left(\gamma_{1} c(L, \rho)\right)^{n} \geq \hat{\Psi}$, which implies

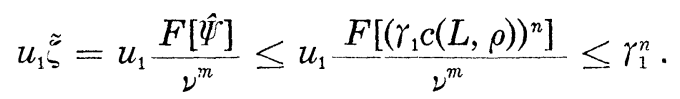

Hence

$$
\zeta^{1 / n} \nu^{m} \leq \frac{\gamma_{1}}{u_{0}} \nu^{m}=\eta \gamma_{1} f^{*}(c(L, \rho)) \wedge \nu^{m-1}
$$

It follows from Lemma 7 that

$$
\begin{aligned}
\|\left._{\varepsilon} B\left(t, \frac{\zeta}{\eta^{n}}\right)\right|_{s} ^{r} & =n B\left(r, \zeta^{1 / n}(\eta \gamma)^{-1}\right)+\frac{c}{2} \log \gamma_{1}^{n}-B\left(s, \frac{\zeta}{\eta^{n}}\right) \\
& \leq \frac{n c}{2}\{(1+2 \varepsilon) \log T(r, s)+5 \varepsilon \log r\} \leq \frac{p}{2 k} T(r, s)+3 n c \varepsilon \log r
\end{aligned}
$$

if $r$ is large enough. Therefore

$$
\begin{aligned}
\|_{\varepsilon} \mathfrak{n} N\left(r, s ; D_{f}\right)+\frac{p}{2 k} T(r, s) \leq & \operatorname{Ric}_{\tau}(r, s)-S_{f}(r, s)+\left.\mathfrak{n} B(t, \eta)\right|_{s} ^{r} \\
& +3 n c \varepsilon \log r .
\end{aligned}
$$

Now (35) and (42) yield (9).

q.e.d.

Remark. If $F$ be dominated by $\tau$ with $Y$ as dominator, i.e.

$$
n\left(\frac{F\left[\psi^{n}\right]}{\nu^{m}}\right)^{1 / n} \nu^{m} \leq Y(r) f^{*}(\psi) \wedge \nu^{m-1} \quad \text { on } M[r]
$$

holds for all continuous form $\psi \geq 0$ of bidegree $(1,1)$ on $M$, which implies

$$
n\left(\frac{u_{0}^{n}}{u_{1}}\right)^{1 / n} \eta \leq Y(r)
$$

Then

$$
S_{f}(r, s) \geq-\mathfrak{n} N\left(r, s ; D_{f}\right)-\frac{n c}{2} \log \frac{Y(r)}{n}+\left.\mathfrak{n} B(t, \eta)\right|_{s} ^{r} .
$$

Hence (42) and (43) yield

$$
\|_{\varepsilon} \frac{p}{2 k} T(r, s) \leq \operatorname{Ric}_{\imath}(r, s)+\frac{n c}{2} \log \frac{Y(r)}{n}+3 n c \varepsilon \log r
$$


which is the (4) in Theorem C.

Proof of Corollary 2. By Stoll [8], there exist effective Jacobian sections of $f$ and holds the following

$$
0 \leq \lim _{r \rightarrow \infty} \frac{\operatorname{Ric}_{\tau}(r, s)}{\log r}<\infty .
$$

Then the condition (ii) and Theorem 1 imply

$$
A(\infty)=\lim _{r \rightarrow \infty} A(r)=\lim _{r \rightarrow \infty} \frac{T(r, s)}{\log r}<\infty
$$

where $A(r)=A\left(r, f^{*} c(L, \rho)\right)$. Hence $f$ is rational (see [8]).

q.e.d.

Remark. The condition (ii) can be replaced by

$$
E_{f}=\varlimsup_{r \rightarrow \infty} \frac{E_{f}(r, s)-\mathfrak{n} N\left(r, s ; D_{f}\right)}{\log r}<\infty .
$$

If $M$ is smooth affine algebraic variety with $m \geq n$, then there exists an effective Jacobian section of $f$ and dominated by $\tau$ with a constant dominator $Y=m$. It follows from (35) and (43) that

$$
\varlimsup_{r \rightarrow \infty} \frac{E_{f}(r, s)-n N\left(r, s ; D_{f}\right)}{\log r} \leq \varlimsup_{r \rightarrow \infty} \frac{(1-n) \operatorname{Ric}_{\tau}(r, s)}{\log r} \leq 0 .
$$

Hence (ii)' holds for this case and Theorem B follows from Corollary 2.

Remark. If $M=C^{m}$, then $\operatorname{Ric}_{\tau}(r, s)=0$ where $\tau$ is defined by $\tau(z)=$ $|z|^{2}$. Now (9) yields

$$
E_{f} \geq c_{1} A(\infty)>0
$$

because the line bundle $L$ is positive and $\operatorname{rank} f=b$. Hence we have

COROLlary 8. Let $N$ be a connected, n-dimensional projective algebraic manifold of general type. Then any holomorphic mappings $f: C^{m} \rightarrow N$ with $E_{f} \leq 0$ has everywhere rank less than $\min (m, n)$.

Theorem A follows from Corollary 8 and Remark above.

Remark. If $\psi$ satisfies

$$
\varlimsup_{r \rightarrow \infty} \log T\left(r, s ; f^{*}(\psi)\right) / T(r, s)=0
$$

by the proof of Theorem 1, Theorem 1 holds for such $\psi$. 


\section{REFERENCES}

[1] S. S. Chern, Shiing-Shen Chern selected papers, Springer-Verlag New York Heidelberg Berlin, 347-360.

[2] Ph. Griffiths, Holomorphic mapping into canonical algebraic varieties, Ann. of Math., (2) 93 (1971), 439-458.

[ 3 ] Ph. Griffiths and J. King, Nevanlinna theory and holomorphic mappings between algebraic varieties, Acta. Math., 130 (1973), 145-220.

[4] P. C. Hu, On Griffiths' Conjecture in value distribution of holomorphic maps, to appear.

[5] S. Kobayashi and T. Ochiai, Mappings into compact complex manifolds with negative Chern class, J. Math. Soc. Japan, 23 (1971), 137-148.

[6] K. Kodaira, On holomorphic mappings of polydises into compact complex manifolds, J. Diff. Geom., 6 (1971), 33-46.

[7] R. Nevanlinna, Eindeutige analytische Funktionen. Die Grundl., d. Math. Wiss. XLVC Springer Verlag. Berlin-Göttingen-Heidelberg 2 ed. 1953.

[ 8 ] W. Stoll, Value distribution on parabolic spaces. Lecture Notes in Mathematics 600 Springer-Verlag, Berlin-Heidelberg-New York. 1977.

[9] - Value distribution theory for meromorphic maps. Aspects of Mathematics, Vieweg. 1985.

Department of Mathematics

Shandong University

Jinan, Shandong,

China 